\title{
SIBRAGEC

\section{APLICAÇÃO DA METODOLOGIA RASE NA NORMA DE ACESSIBILIDADE ASSOCIADA AO BIM ${ }^{1}$}

\author{
SANTOS, Kathleen Tácila (1); SAMPAIO, Marco Antônio Brasiel (2) \\ (1) Universidade Federal de Sergipe, kathleentacila@gmail.com, (2) Universidade Federal de \\ Sergipe,marco.sampaio@ufs.br
}

\begin{abstract}
RESUMO
A metodologia Building Information Modeling (BIM) tem se tornado essencial nos últimos anos para a indústria da Arquitetura, Engenharia e Construção (AEC). Dentre os seus principais usos aplicados à Construção Civil, a verificação automatizada de normas tem ganhado destaque na sua utilização, especialmente pelo fluxo de informações utilizados na metodologia. A redução de fatores como imprecisões de análises, tempo prolongado e interpretações pessoais tornam-se imprescindíveis para a garantia da qualidade do empreendimento, sobretudo na fase de projetos, onde o uso é aplicado. Considerando a sua importância, o presente artigo apresenta a aplicação da verificação automática a partir da metodologia RASE na Norma de Acessibilidade, a ABNT NBR 9050:2020. Além das recomendações da literatura, utilizou-se um modelo BIM, a fim de aplicar a programação visual, desenvolvidos em softwares específicos. Dentre os principais resultados destacam-se a aplicação da metodologia RASE na norma brasileira, o desenvolvimento da rotina de programação e a verificação automática, gerando resultados visuais diretamente no modelo. Além dos ganhos de tempo, redução de erros e melhoria na qualidade do projeto, a metodologia RASE, quando aplicada a este uso BIM, potencializa-o, tornando-o ainda mais acessível e aplicável à comunidade.
\end{abstract}

Palavras-chave: Verificação automatizada, Verificação de regras, BIM, Norma de Acessibilidade.

\begin{abstract}
The Building Information Modeling (BIM) methodology has become essential in the last years for the Architecture, Engineering and Construction (AEC) industry. Among its main uses applied to Civil Construction, automated standards verification has gained prominence in its use, especially due to the flow of information used in the methodology. The reduction of factors such as analysis inaccuracies, prolonged time and personal interpretations become indispensable for the quality warranty of the enterprise, especially in the project phase, where the use is applied. Considering its importance, this article presents the application of the automatic verification based on the RASE methodology in the Accessibility Standard, ABNT NBR 9050: 2020. In addition to the literature recommendations, a BIM model was used in order to apply the visual programming, developed in specific softwares. Among the main results, stand out the application of the RASE methodology in the Brazilian standard, the development of the programming routine and the automatic verification, generating visual results directly in the model. In addition to time savings, error reduction and improvement in the quality of the project, the RASE methodology, when applied to this BIM use, potentiates it, making it even more accessible and applicable to the community.
\end{abstract}

Keywords: Automated verification, Code Checking, BIM, Accessibility Standard.

\section{INTRODUÇÃO}

O Building Information Modeling (BIM) adentra o mercado da construção civil para revolucionar as atividades e desenvolvê-las com a maior garantia de qualidade do

\footnotetext{
${ }^{1}$ SANTOS, K. T.; SAMPAIO, M. B. Aplicação da metodologia RASE na norma de acessibilidade associada ao BIM. In: SIMPÓSIO BRASILEIRO DE GESTÃO E ECONOMIA DA CONSTRUÇÃO, 12. 2021, Maceió. Anais[...] Porto Alegre: ANTAC, 2021. p.1-8. Disponível em:

https://eventos.antac.org.br/index.php/sibragec/article/view/500. Acesso em: 2 out. 2021.
} 
produto. Dentre as diversas utilidades da plataforma, a verificação de regras torna-se uma grande aliada, sobretudo quando se trata das atividades relacionadas à verificação de normas específicas, leis municipais ou locais e demais vertentes similares.

Atualmente, o analista utiliza de meios manuais e mecânicos para verificar os projetos, utilizando-se do seu conhecimento próprio e das regras exigidas, cuja verificação é feita a partir da análise do projeto através de seus componentes e relações. No entanto, como esta análise é feita por diferentes analistas, nem sempre é possível garantir que haja um padrão e, por conseguinte, uma garantia da qualidade do produto, tornando a análise suscetível à propagação de erros.

Segundo Solihin e Eastman (2015), existem duas vertentes principais que devem ser levadas em consideração para o processo de verificação: a primeira é o modelo de construção BIM, enquanto a segunda é definida pelo conjunto de regras que será utilizado para a análise. No entanto, é sabido que os projetos são, na verdade, grandes conjuntos de dados e, até mesmo para estes, não se torna possível definir um conjunto padrão que seja aplicado a todos os conjuntos de dados, tornando-se necessária a aplicação de conjunto de regras para um subconjunto de dados.

De acordo com Hjelseth e Nisbet (2011), há na bibliografia duas razões principais para a limitação dos estudos na verificação de regras automatizadas. A primeira diz respeito ao fato de que nem todos os modelos de normas de construção são capazes de representar todos os tipos de regras; a segunda, por sua vez, está relacionada à dificuldade de manter as normas, uma vez que os modelos estão sujeitos à mudança contínua das regras existentes e da adição de novas. Essas limitações tornaram-se objetos de estudo de caso para o presente artigo.

Hjelseth e Nisbet (2010) demonstraram que as regras de uma norma podem ser especificadas a partir da interpretação semântica do texto, com o uso de quatro operadores que dão base à metodologia RASE: requerimento, aplicabilidade, seleção e exceção. Apesar do êxito em todas as validações feitas pelos autores, eles afirmam que não é possível considerá-las uma evidência científica visto que, dentre outros fatores, textos mal estruturados e pouco claros podem levar a problemas no desenvolvimento das regras. Assim, sugere-se que as normas locais devem ser avaliadas quanto à aplicação da metodologia. Neste sentido, o presente trabalho apresenta como objetivo analisar a aplicação da metodologia RASE na Norma de Acessibilidade, NBR 9050:2020.

\section{REFERENCIAL TEÓRICO}

\subsection{Estrutura da norma}

A Norma Brasileira de Acessibilidade, NBR 9050:2020, estabelece as especificidades técnicas que devem ser obedecidas para proporcionar melhores condições de mobilidade, bem-estar e segurança para os indivíduos portadores de necessidades especiais, seja por mobilidade, percepção ou outra limitação.

As normas de construções, de uma forma geral, podem ser apresentadas de forma textual ou gráfica. Macit et al. (2013) classifica, ainda, a forma textual em três tipos, são elas: geral, que descreve o objetivo, escopo e base legal do código de construção; as declarações de definição, que dá detalhes descritivos acerca dos códigos; e as declarações de regra, que apresentam as restrições sobre os componentes, espaços e relações de construção física.

Considerando a automatização de verificação de normas, observa-se que cada item da norma possui algum grau de complexidade em sua implementação, podendo ser uma simples verificação explícita ou verificações que envolvem relações complexas entre 
diferentes elementos do projeto. Nesse sentido Solihin e Eastman (2015) definem quatro classes distintas que diferenciam os graus de complexidade na verificação, conforme mostrado no Erro! Fonte de referência não encontrada..

Quadro 1. Classificação de regras para a verificação.

\begin{tabular}{|c|c|c|}
\hline Classe & Descrição & Complexidade \\
\hline 01 & $\begin{array}{c}\text { Informações existentes explicitamente no pacote } \\
\text { de dados do modelo BIM }\end{array}$ & $\begin{array}{c}\text { Dados simples ou obtidos de } \\
\text { associações explícitas }\end{array}$ \\
\hline 02 & $\begin{array}{c}\text { Informações obtidas a partir de derivações } \\
\text { implícitas, mas que não geram novas estruturas de } \\
\text { dados através das derivações }\end{array}$ & $\begin{array}{c}\text { Informação não contida no elemento a } \\
\text { ser analisado, geralmente gerada por } \\
\text { meio de cálculos trigonométricos ou } \\
\text { aritméticos }\end{array}$ \\
\hline 03 & $\begin{array}{c}\text { Checa as informações a partir de variáveis } \\
\text { complexas, gerando novas estruturas de dados }\end{array}$ & $\begin{array}{c}\text { Informação não contida no elemento a } \\
\text { ser analisado, gerada através de } \\
\text { derivações complexas que envolvem a } \\
\text { definição de novos elementos }\end{array}$ \\
\hline 04 & $\begin{array}{c}\text { Destinada aos itens que requerem uma "prova de } \\
\text { solução" ou que podem ser modificados para } \\
\text { atender a certas verificações }\end{array}$ & $\begin{array}{c}\text { Semelhante às outras classes, com } \\
\text { requisitos diferenciados de informações } \\
\text { básicas para gerar soluções }\end{array}$ \\
\hline
\end{tabular}

Fonte: Autores, 2021.

\subsection{Abordagem semântica}

A Metodologia RASE (Requirement, applicability, selection, exception) surgiu pela necessidade de gerenciamento de dados, uma vez que uma única planilha não seria capaz de suportar a quantidade de informações geradas pelos modelos. Ela faz parte do conjunto de métodos usados para transformar as normas textuais em dados lógicos. Consiste, portanto, em um modelo semântico de quatro marcadores de operação: requisito, aplicabilidade, seleção e exceção.

É sugerido por Hjelseth e Nisbelt (2010) que se aplique a sequência: seleção, aplicabilidade, requerimento e exceção. Isso se dá porque o operador seleção abrange o tópico no todo, enquanto a aplicabilidade se considera a um item específico deste. $\mathrm{O}$ operador de requerimento e exceção aplica-se diretamente às condições que são requeridas e as excludentes, respectivamente. Estas características podem ser compreendidas a partir do

, que apresenta exemplos de cada operador, a partir do item 6.7 e subitem 6.7.1.

Quadro 2. Descrição e exemplificação da Metodologia RASE.

\begin{tabular}{|c|c|c|}
\hline Operador & Descrição & Exemplo \\
\hline Seleção (Selection) & Elemento generalizado & Escada \\
\hline Aplicabilidade (Applicability) & Elemento específico & da escada \\
\hline Requisito (Requirement) & $\begin{array}{c}\text { Regra de compreensão e } \\
\text { obtenção direta }\end{array}$ & $\begin{array}{c}\text { Projeção da aresta do bocel menor } \\
\text { ou igual a } 1,50 \mathrm{~cm}\end{array}$ \\
\hline Exceção (Exception) & $\begin{array}{c}\text { Elemento dado como exceção, } \\
\text { obtido a partir de expressóes }\end{array}$ & Emeíveis \\
\hline
\end{tabular}

Fonte: Autores (2011). 


\section{METODOLOGIA}

O presente estudo pode ser caracterizado como descritivo, uma vez que analisa os dados obtidos e suas respectivas variáveis de estudo. Quanto à coleta de dados, considera-se o caráter documental, uma vez que trabalha com fontes secundárias, como teses e artigos confiáveis publicados na área de estudo. Além disso, a pesquisa é caracterizada como um estudo de caso, pela aplicação direta do levantamento de informações obtido e a geração de análise a partir deste.

A fim de atender ao objetivo proposto, fez-se uma revisão na literatura, considerando as principais publicações acerca da aplicação da verificação automática de normas em modelos BIM e da metodologia RASE.

O escopo geral da análise de dados da pesquisa pode ser dividido em quatro etapas: classificação dos itens da NBR 9050; tradução da norma para um formato de dados; programação e modelagem; e, por fim, a aplicação.

A primeira etapa utilizou as quatro classes propostas por Solihin e Eastman (2015) para agrupar os itens da norma. No presente trabalho, somente os itens da Classe 1 são utilizados para a verificação automática. Aplicando os conceitos do RASE aos itens selecionados, estes puderam ser traduzidos para um modelo lógico em formato JSON.

A metodologia foi implementada em linguagem de programação visual com o Dynamo ${ }^{\circledR}$, associado a um modelo BIM desenvolvido no aplicativo Autodesk Revit $2020^{\circledR}$. O modelo se trata de um salão comercial hipotético com banheiro social, escritório, área de ventilação, rampa e estacionamento, conforme pode ser visualizado na Erro! Fonte de referência não encontrada. .

Figura 1. Representação do modelo utilizado para a verificação.

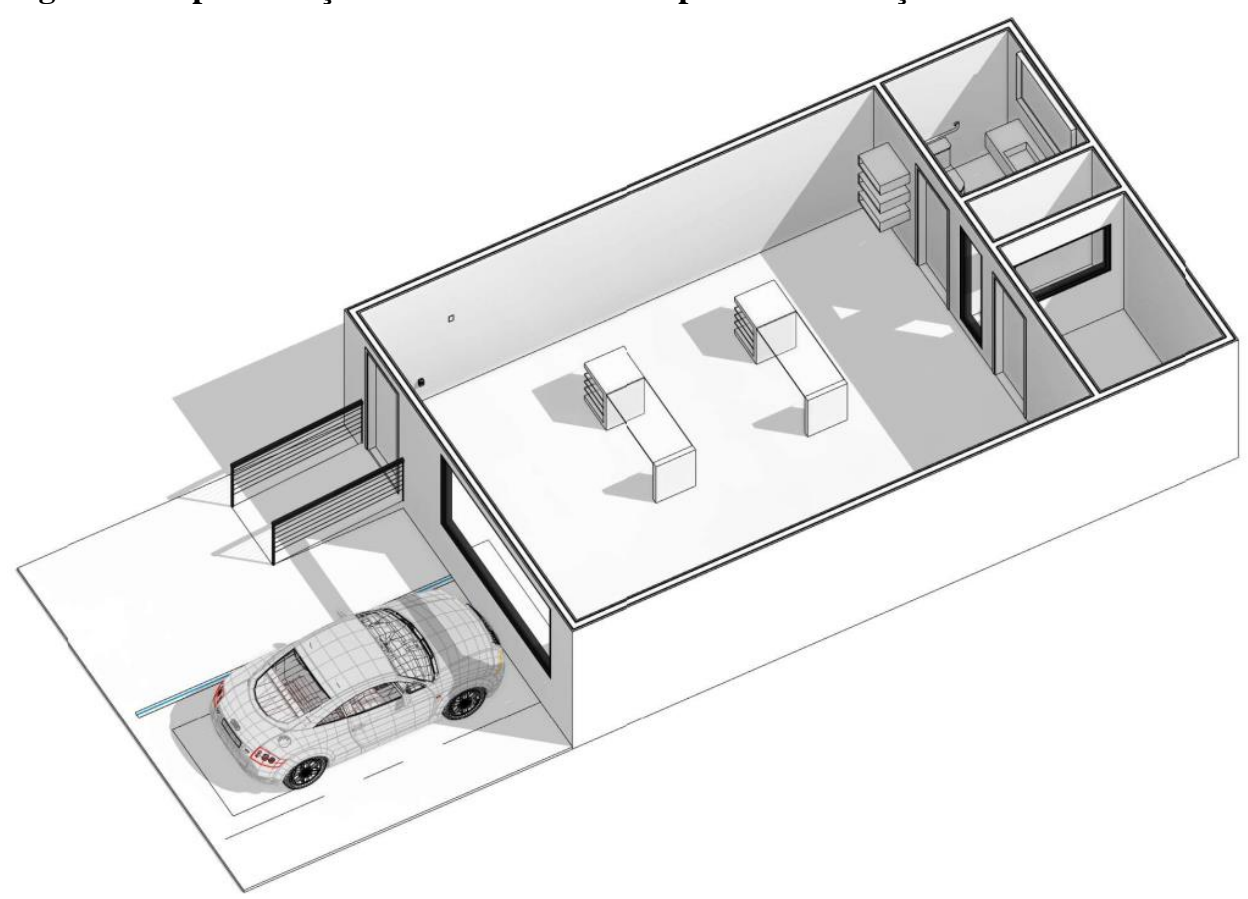

Fonte: Autores, 2021.

Para a transcrição da norma em regras verificáveis computacionalmente, levou-se em consideração três itens da Norma: item 6 (acessos e circulações), item 7 (sanitários, banheiros e vestiários) e item 8 (mobiliário urbano).

É importante considerar que nem todas as informações apresentadas pela norma são passíveis de transcrições devido à subjetividade, em menor ou maior grau. Por isso, fezse um levantamento dos três itens citados acima levando-se em consideração as regras 
explícitas, separando-as em recomendações normativas e classificando-as conforme a complexidade da regra. É possível observar esse tipo de texto na norma, tal qual o item 6.1.2 (2020, p. 52) que infere a altura da iluminação numa rota acessível: "Toda rota acessível deve ser provida de iluminação natural ou artificial com nível mínimo de iluminância de 150 lux medidos a 1,00 m do chão.”.

De forma análoga, classificou-se como implícitos os códigos que geraram ambiguidades ou que se apresentaram completamente subjetivo. Esse tipo de texto é possível observar no item 6.2.7, que diz respeito à existência ou não de portas giratórias e suas recomendações.

\begin{abstract}
Quando existir porta giratória, deve ser prevista, junto a esta, outra entrada que garanta condições de acessibilidade. Portas giratórias devem ser evitadas, mas quando forem instaladas, as dimensões entre as pás devem ser compatíveis com as medidas necessárias para o deslocamento de uma pessoa em cadeira de rodas e devem ainda ser dotadas de sistema de segurança para rebatimento das pás em caso de sinistro. (ABNT, 2020, p. 53)
\end{abstract}

A sequência de operação da rotina elaborada no Dynamo consistiu em quatro etapas: entrada de dados, aplicação do RASE, subprocessos e a saída de dados.

A entrada de dados é composta por nós que leem o arquivo json. As informações são extraídas e conduzidas aos nós agrupados representando cada operador. Baseado nestes dados, o operador seleção obtêm todos os elementos potenciais e os passam para o grupo do operador de aplicabilidade, que separa os elementos que se enquadram no critério de verificação. No terceiro operador, o requisito, os parâmetros desses elementos são verificados. De modo similar, o operador de exceção elimina os elementos não condicionados.

Para cada verificação é criada uma lista contendo o número de identificação (id) e a condição de verificação, isto é, V ou F. A posterior, os elementos iguais são agrupados para permitir a contagem dos checks que passam ou não em todas as verificações a que foi submetida. Por fim, a última etapa é composta de um relatório visual, que modifica as cores dos objetos no modelo, de modo que os elementos se tornam verde se estes apresentarem-se de modo correto e vermelho, se não. Essa rotina pode ser melhor observada por meio do fluxograma representado na Figura 2.

Figura 2. Fluxograma da rotina utilizada para a programação visual.

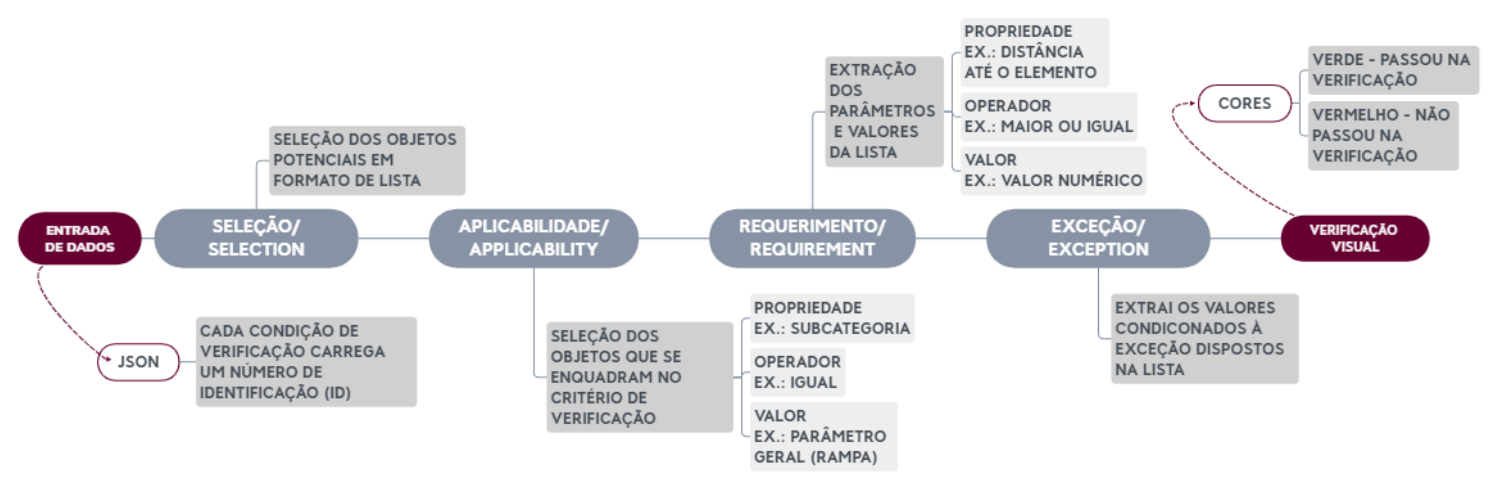

Fonte: Autores (2021).

\title{
4 RESULTADOS E DISCUSSÕES
}

Diante dessas informações, conseguiu-se obter, ao todo, 238 recomendações normativas, sendo 116 para o item 6, 101 para o item 7 e 21 para o item 8 da respectiva norma. É importante ressaltar uma pequena diferença em relação ao método proposto por Hjelseth 
e Nisbet (2010), que utiliza o formato XML. Apesar disso, nenhuma mudança conceitual é esperada, uma vez que se trata de um meio de armazenamento de dados. O processo de automatização iniciou com a tradução da norma para um formato de dados, armazenado em formato JSON. O algoritmo principal realiza três funções principais: gerenciamento da metodologia RASE, leitura dos parâmetros da norma e interação com o modelo BIM.

Para cada uma das recomendações, obteve-se a classificação das regras, conforme descrito no Quadro 3. Algumas recomendações apresentaram mais de um parâmetro para ser verificado. Vale ressaltar que a análise foi elaborada para a utilização do software Revit, uma vez conhecidos seus parâmetros e informações que poderiam ser extraídos.

Quadro 3. Classificação das regras e recomendações por itens normativos.

\begin{tabular}{|c|c|c|c|c|c|}
\hline & Recomendações Normativas & Classe 01 & Classe 02 & Classe 03 & Classe 04 \\
\hline Item 6 & 116 & 24 & 63 & 28 & 1 \\
\hline Item 7 & 101 & 33 & 58 & 9 & 1 \\
\hline Item 8 & 21 & 8 & 10 & 3 & 0 \\
\hline
\end{tabular}

Fonte: Autores, 2021.

Algumas recomendações, embora estivessem dentro da filtragem de transcrição, não foram passíveis de classificação por limitação do software. A partir das características obtidas da metodologia citada anteriormente, pode-se aplicá-la às regras traduzidas da NBR 9050. Esta caracterização foi feita de modo que, tendo conhecimento do item selecionado, agrupou-se nos operadores, quando houvesse relação. Para facilitar a leitura da rotina, quando a regra exigia um intervalo de dados, separou-se em identificações diferentes, conforme o exemplo explicitado nos Quadro 4Quadro 5.

Quadro 4. Parte I - Aplicação do RASE ao item 6.9.3.2 da NBR 9050:2020.

\begin{tabular}{|c|c|c|c|c|c|}
\hline Texto da Regra & Tópico & Propriedade & Comparador & Valor & Unidade \\
\hline \multirow{8}{*}{$\begin{array}{l}\text { Os corrimãos devem ser } \\
\text { instalados em rampas e } \\
\text { escadas em ambos os } \\
\text { lados, a } 0,92 \text { m e a } 0,70 \mathrm{~m} \\
\text { do piso, medidos da face } \\
\text { superior até o bocel ou } \\
\text { quina do degrau (no caso } \\
\text { de escadas) ou do patamar, } \\
\text { acompanhando a } \\
\text { inclinação da rampa, } \\
\text { conforme Figura } 76 .\end{array}$} & \multicolumn{5}{|c|}{ Selection } \\
\hline & Element & Category & Equal & Railings & Null \\
\hline & \multicolumn{5}{|c|}{ Applicability } \\
\hline & Element & Sub-Category & Equal & Top Rails & Null \\
\hline & \multicolumn{5}{|c|}{ Requeriment } \\
\hline & Parameter & Railing Height & LessEqual & 0,92 & $\mathrm{~m}$ \\
\hline & \multicolumn{5}{|c|}{ Exception } \\
\hline & Null & Null & Null & Null & Null \\
\hline
\end{tabular}

Fonte: Autores (2021).

Quadro 5. Parte II - Aplicação do RASE ao item 6.9.3.2 da NBR 9050:2020.

\begin{tabular}{|c|c|c|c|c|c|}
\hline Texto da Regra & Tópico & Propriedade & Comparador & Valor & Unidade \\
\hline \multirow{8}{*}{$\begin{array}{l}\text { Os corrimãos devem ser } \\
\text { instalados em rampas e } \\
\text { escadas em ambos os } \\
\text { lados, a } 0,92 \mathrm{~m} \text { e a } 0,70 \mathrm{~m} \\
\text { do piso, medidos da face } \\
\text { superior até o bocel ou } \\
\text { quina do degrau (no caso } \\
\text { de escadas) ou do patamar, } \\
\text { acompanhando a } \\
\text { inclinação da rampa, } \\
\text { conforme Figura } 76 .\end{array}$} & \multicolumn{5}{|c|}{ Selection } \\
\hline & Element & Category & Equal & Railings & Null \\
\hline & \multicolumn{5}{|c|}{ Applicability } \\
\hline & Element & Sub-Category & Equal & Top Rails & Null \\
\hline & \multicolumn{5}{|c|}{ Requeriment } \\
\hline & Parameter & Railing Height & GreaterEqual & 0,70 & $\mathrm{~m}$ \\
\hline & \multicolumn{5}{|c|}{ Exception } \\
\hline & Null & Null & Null & Null & Null \\
\hline
\end{tabular}


Embora sejam trabalhados apenas os elementos classificados como nível 01, isto é, simples e de fácil extração, foram identificados alguns empecilhos para a execução da verificação. Estes referem-se à modelagem e a parametrização utilizada, uma vez que não foi feita a padronização completa. Por isso, é necessário que haja uma padronização da modelagem, especialmente nos elementos que devam ser verificados, a partir de um Model View Definition (MVD) bem definido.

\section{CONSIDERAÇÕES FINAIS}

Embora seja um uso da metodologia BIM pouco explorado, a verificação automatizada das normas é essencial para o rápido e assertivo desenvolvimento dos projetos. No entanto, atualmente, esse objetivo é obtido por meio de plataformas pagas, a partir de programação densa. Por essa razão, a metodologia RASE torna-se uma alternativa para tornar o uso acessível aos profissionais da AEC.

Assim, é possível inferir que a abordagem semântica utilizada neste trabalho permite realizar a verificação de normativas por meio da transcrição e tradução das regras. A validação desta metodologia por meio da programação visual corroborou com resultados práticos, uma vez que a utilização do relatório visual permite a fácil identificação dos erros e a posterior modificação.

No entanto, é importante ressaltar as barreiras que podem ser trabalhadas para garantir maior eficiência na automatização desta verificação. A padronização dos elementos por meio de um MVD é essencial para garantir que os elementos permaneçam no nível 01 ou, se este for ausente, será necessário utilizar-se de outras informações para extrair esses dados, fazendo com que as recomendações elevem os seus níveis. Por fim, recomenda-se a utilização dos parâmetros IFC, ainda que não se utilize o MVD, para que, dentro da rotina, obtenha-se os resultados independentes da plataforma utilizada, sendo este comum aos softwares.

\section{REFERÊNCIAS}

ABNT. ASSOCIAÇÃO BRASILEIRA DE NORMAS TÉCNICAS. NBR-9050: Acessibilidade a edificações, mobiliário, espaços e equipamentos urbanos. Rio de Janeiro, 2020.

HJELSETH, E.; NISBET, N. Capturing normative constraints by use of the semantic mark-up rase methodology. In: 28th International Conference-Applications of IT in the 12AEC Industry, Sophia Antipolis, 2011. Proceedings... Sophia Antipolis, France, 2011.

HJELSETH, E.; NISBET, N. Exploring Semantic Based Model Checking. In: 27th CIB W78 International Conference, Cairo, 2010. Proceedings... Cairo, Egypt, 2010.

MACIT, S. et al. İzmir municipality housing and zoning code analysis and representation for compliance checking. In: 20th Workshop of the European Group for Intelligent Computing in Engineering, Vienna, Austria, 2013.

SOLIHIN, W.; EASTMAN, C. Classification of rules for automated BIM rule checking development. Automation In Construction, [S.L.], v. 53, p. 69-82, maio 2015. Elsevier BV. http://dx.doi.org/10.1016/j.autcon.2015.03.003. 